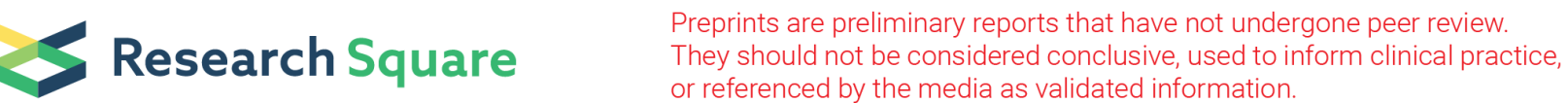

\section{Increased Portal Vein Diameter is Predictive of Portal Vein Thrombosis Development in Patients with Liver Cirrhosis}

\section{Gang Dong}

Zhongshan Hospital Fudan University

\section{Xiaoquan Huang}

Zhongshan Hospital, Fudan University

\section{Yuli Zhu}

Zhongshan Hospital, Fudan University

\section{Hong Ding}

Zhongshan Hospital, Fudan University

\section{Feng Li}

Zhongshan Hospital, Fudan University

Shiyao Chen ( $\square$ chen.shiyao@zs-hospital.sh.cn )

Zhongshan Hospital, Fudan University

\section{Research}

Keywords: cirrhosis, thrombosis, portal vein diameter

Posted Date: June 15th, 2020

DOI: https://doi.org/10.21203/rs.3.rs-35144/v1

License: () (1) This work is licensed under a Creative Commons Attribution 4.0 International License. Read Full License

Version of Record: A version of this preprint was published at Annals of Translational Medicine on February 1st, 2021. See the published version at https://doi.org/10.21037/atm-20-4912. 


\section{Abstract}

Background: Cirrhotic patients with portal vein thrombosis (PVT) may have a high risk of hepatic decompensation and increased mortality. This study aimed to investigate if increased portal vein diameter is associated with PVT development.

Methods: A total of 174 cirrhotic patients were enrolled between February 1 and August 31, 2017. All participants were divided into PVT $(n=62)$ and non-PVT $(n=112)$ groups based on the thrombus that was detected by ultrasonography and confirmed by computed tomography angiography (CTA).

Results: The study participants, aged $54.7 \pm 10.5$ years (PVT) and $55.8 \pm 11.6$ years (non-PVT), were included in this analysis. The Child-Pugh score of PVT or non-PVT was $6.6 \pm 1.3$ and $5.8 \pm 0.9$, respectively. Hepatitis B virus (HBV) is the primary etiological agent of cirrhosis. Logistic regression, receiver operating characteristic (ROC), and nomograph analysis designated portal diameter as the strongest independent risk factor for predicting PVT development (odds ratio (OR): 3.96 , area under the ROC curve (AUC): $0.88 ; P<0.01)$, and the cutoff with predictive value for PVT development was $>12.5 \mathrm{~mm}$. No differences were observed in the overall survival (OS) in cirrhosis with or without PVT or stratifying on portal diameter based on the cutoff value.

Conclusions: Increased portal diameter is associated with an increased risk of PVT development. Patients with cirrhosis and increased portal diameter are a high-risk subgroup that may need thromboprophylaxis.

\section{Introduction}

Portal vein thrombosis (PVT) is a major complication in patients with liver cirrhosis and $<1 \%$ in the general population. However, $0.6-15.8 \%$ of the cirrhosis patients e suffered from PVT, varied prevalence PVT according to different studies [1]. Several studies showed that PVT was associated with poor outcomes of cirrhosis patients, such as increasing hepatic decompensation, transplantation mortality, and hepatic artery thrombosis [2-4].

The potential mechanism of the development of PVT in cirrhosis patients is not yet clarified, and the occurrence of PVT is a multifactorial process involving inherited and acquired thrombotic risk factors [5]. Cirrhosis and portal hypertension induced hepatic structural derangement, slowed portal vein blood flow, damaged the vessel wall, and induced hypercoagulability, which served as vital roles in PVT development $[6,7]$.

PVT is primarily detected and diagnosed by imaging examination, especially computed tomography angiography (CTA) [8]. However, due to the high cost and damage to the body, CTA is not suitable for high-risk population screening. Thus, ultrasonography is widely used for screening of high-risk populations with PVT with a diagnostic accuracy of $88-98 \%$ [9]. This method is economical, practical, portable, and harmless, and some parameters of the portal vein, such as portal vein diameter, blood flow velocity, and filling defect, could be measured. The blood flow velocity in the portal vein has been reported 
to predict PVT development [7]. However, the effect of portal vein diameter on the formation of PVT in cirrhotic patients is largely unknown. Studies have demonstrated that portal vein dilatation tends to be continuous in cirrhosis [10]. Then, the dilated portal vein induces endothelial cell damage [11], which is one of the key factors for thrombosis. Nonetheless, only a few reports are available about the association between portal diameter and PVT development in liver cirrhosis. Therefore, the present study aimed to evaluate the effect of portal diameter on PVT development and prognosis in cirrhotic patients.

\section{Materials And Methods}

\section{Patients}

From February-August in 2017, 174 patients diagnosed with liver cirrhosis, screened by CT or magnetic resonance (MR), and underwent ultrasonography of the portal venous system, were enrolled. The exclusion criteria were as follows: (1) malignant tumor; (2) active hemorrhage; (3) acute infections; (4) receiving antiplatelet or anticoagulant therapy; (5) a history of abdominal surgery including splenectomy. Subsequently, the patients were divided into the PVT and non-PVT groups based on CTA. All participants were followed up until April 2020. This study protocol complied with the guidelines of the Declaration of Helsinki and was approved by the Medical Ethics Committee of Zhongshan Hospital, Fudan University, China.

\section{Blood collection and analysis}

Blood samples were withdrawn after $12 \mathrm{~h}$ of overnight fasting. Platelets count, D-dimer, total cholesterol, triglyceride, and LDL levels, and other biochemical parameters, such as hepatic function, were measured.

\section{Ultrasound with hepatic portal doppler}

After fasting for $>8 \mathrm{~h}$, participants lay supine, breathed normally, kept quiet, and underwent color Doppler ultrasonic diagnosis. The angle between the portal vein and ultrasonic beam was $<60^{\circ}$, and all measurements were conducted using a 2.0-5.0 MHZ convex transducer (iU22Ultrasound System; Philips Healthcare, Reedsville, PA, USA). The diameters and velocities of the portal vein were measured using Btype ultrasonography and Doppler examination, respectively.

\section{Statistical analysis}

Continuous variables are reported as a mean \pm standard deviation, and categorical data are expressed as numbers and proportions. Statistical analysis was performed using Student's unpaired t-test or MannWhitney test for comparisons of continuous variables between groups and corrected chi-square test or Fisher's exact test was used for categorical data. Logistic regression models were constructed to assess 
risk factors for the development of PVT. Kruskal-Wallis test was used to identify the correlation between portal diameter and etiology or thrombus range, respectively. The sensitivity and specificity of risk factors in predicting PVT were evaluated by receiver operating characteristic (ROC) curves, and the risk was assessed by a nomograph. The Kaplan-Meier analysis was used to estimate the rate of overall survival (OS) in patients with and without PVT according to stratified portal diameter. $\mathrm{P}<0.05$ was considered statistically significant. All analyses were performed using SPSS 24.0 (SPSS Inc., Chicago, IL, USA).

\section{Results}

\section{Participant Characteristics}

The mean age of the study participants was $54.7 \pm 10.5$ years (PVT) and $55.8 \pm 11.6$ years (non-PVT). The cohort consisted of $59.7 \%$ males in PVT and $60.7 \%$ males in non-PVT). Among these patients, hepatitis B virus (HBV) is the major cause of cirrhosis (PVT $59.7 \%$ vs. non-PVT 57. 1\%). The Child-Pugh score of PVT and non-PVT was $6.6 \pm 1.3$ and $5.8 \pm 0.9(P=0.004)$. As shown in Table 1, platelet count and D-dimers examined in the laboratory were $128 \pm 94.3$ (PVT), 79.5 \pm 52.8 (non-PVT) and $2.4 \pm 2.9$ (PVT), $0.7 \pm 1.0$ (non$P V T$ ), respectively. Also, total cholesterol (PVT, 3.6 \pm 1.0 ; non-PVT, 3.5 \pm 0.9 ), triglyceride (PVT, $0.8 \pm 0.4$; nonPVT, 0.9 \pm 0.4 ), and LDL levels (PVT, 1.9 \pm 0.8 ; Non-PVT, $1.8 \pm 0.8$ ) were detected. Portal diameter and portal flow detected by ultrasonography were $14.0 \pm 3.0 \mathrm{~mm}$ (PVT), $10.8 \pm 1.1 \mathrm{~mm}$ (non-PVT) and $16.7 \pm 6.9 \mathrm{~cm} / \mathrm{s}$ (PVT), $19.0 \pm 2.9 \mathrm{~cm} / \mathrm{s}$ (non-PVT), respectively, and PVT was distributed in the main portal vein $(72.6 \%)$.

\section{Role of portal diameter on PVT development}

Logistic regression analysis revealed that portal diameter, portal flow, platelet, and D-dimer were identified as independent risk factors for PVT $(P<0.05)$, and the effect of the Child-Pugh score was rendered insignificant (Table 2). The area under the ROC (AUC) of the portal diameter for PVT development was 0.88 (Figure 1A), which was significant among risk factors, and the sensitivity and specificity for portal diameter in predicting PVT development were $63 \%$ and $89 \%$, respectively (Table 3). On the other hand, the AUC of the combination of the above factors for PVT development was 0.91 (Figure 1B), and the sensitivity and specificity for portal diameter in predicting PVT development were $87 \%$ and $95 \%$, respectively (Table 3), which was consistent with the assessment by nomograph (Figure 2). Cirrhosis could result from a variety of etiologies, which has no influence on the portal diameter and was unaffected by the range of thrombus (Figure 3). Based on the cutoff of portal diameter (Table 3), the PVT participants were divided into two groups: $\leq 12.5 \mathrm{~mm}$ and $>12.5 \mathrm{~mm}$. No differences were detected in the OS while classifying according to the portal diameter during follow-up; also, the OS was similar in patients with and without PVT (Figure 4).

\section{Discussion}


In the present study, 174 cirrhosis patients with or without PVT were investigated. The results established a positive association between portal diameter and PVT occurrence in cirrhosis patients, and compared it to portal flow, platelet count, and D-dimer; patients with increased portal diameter may be at a high risk to PVT development. This study also confirmed the cutoff of portal diameter as $>12.5 \mathrm{~mm}$, which was sensitive for discerning the cirrhosis patients at risk for PVT development. Based on the cutoff, Bultrasonography determining the portal diameter should be considered a potential screening tool for identifying patients at the highest risk of thrombosis.

HBV has been identified as the most frequent cause of cirrhosis and considered as a risk factor for PVT $[12,13]$. In this study, the etiology was HBV in cirrhosis patients with or without PVT. In addition, several other risk factors have been reported to play a role in the development of PVT in cirrhosis [14-16]. Since the majority of PVT has no obvious early symptoms, it could be missed diagnosed or be misdiagnosed; subsequently, PVT severely affects the prognosis of cirrhosis patients, including increasing the bleeding risk of varices and liver transplantation mortality [2-4]. Therefore, it is crucial to screen out the most valuable risk factors to predict the early development of PVT. Reportedly, the development of PVT in cirrhosis is multifactorial, such as unbalanced coagulation and anti-coagulation activity contributing towards the hypercoagulable state, blood flow stasis from portal hypertension, synergistic interaction of vessel wall, and endothelial injury [6]. Elevated blood lipid levels are related to venous thrombosis [17]; however, in this study, the correlation between blood lipid levels and PVT was not significant (data not shown), and portal flow, platelet count, Child-Pugh score, D-dimer, and portal diameter were further screened to evaluate their roles in predicting PVT development. As shown in Table 2, an inverse correlation was established between portal flow and PVT occurrence and slow blood flow increased the contact between platelet and vessel wall, which was beneficial to thrombosis, and flow rates $<15 \mathrm{~cm} / \mathrm{s}$ were associated with increased PVT development [7]; nevertheless, the cutoff in this study was 18.5 $\mathrm{cm} / \mathrm{s}$. The other risk factor was platelet count, the association between platelet count and PVT was statistically significant, and the cutoff value was 111.5. Increased platelet indices also contribute to the prethrombotic state in liver cirrhosis and that large platelet may play a specific role in thrombosis [18]. Contrary to the previous report [19], we did not find a significant association between child score and PVT development in the present study. The Child-Pugh score is commonly used in clinical practice for quantitative assessment of liver reserve function in patients with liver cirrhosis; the higher the score, the worse the liver reserve function, and the reported incidence of PVT in advanced liver disease increased up to $40 \%$ [20]. However, this could be ascribed to the Child-Pugh score A or B in the current study population with adequate liver reserve function; hence, our experimental results were inconsistent with previous studies. D-dimer is a major indicator of thrombosis, and detection of D-dimer is primarily used in the prediction and diagnosis of venous thrombosis [21, 22]. in this study, D-dimer was compared to the portal diameter to predict the PVT development. Cirrhosis has been reported to induce continuous portal vein dilation, which would further damage endothelial cells and then promote the formation of thrombus [10, 11]. To date, few relevant clinical studies are available about the association between portal diameter and PVT development in cirrhosis. Herein, we found a significant association between portal diameter and PVT, and AUC was higher than other risk factors, screening high-risk patients with PVT by 
ultrasonography, and intervening early will improve the prognosis of cirrhosis. HBV is one of the risk factors of PVT [6]. However, the portal diameter was not related to the etiology, and the range of PVT involvement had little effect on the portal diameter.

A previous study demonstrated that the mortality in PVT patients was increased [23]; however, in the current study, no differences were detected in the survival time in cirrhosis patients with or without PVT. This phenomenon could be explained by the fact that the majority of the enrolled patients were ChildPugh A or B, and the liver function was compensatory. Additionally, endoscopic treatment to prevent rebleeding and other complications, anticoagulant therapy, and antiviral therapy are critical factors that improve the prognosis of PVT patients. Nevertheless, the limitations of the current study include the small sample size, short follow-up period, and data from a single center. These factors might affect the survival time in cirrhosis patients with or without PVT. Thus, a long-term follow-up of a large sample is essential for an objective evaluation of PVT in the role of the survival of patients with cirrhosis. Moreover, no difference was detected in the survival rates when stratifying portal diameter to those with $\leq 12.5 \mathrm{~mm} v s$. $>12.5 \mathrm{~mm}$ at follow-up.

\section{Conclusions}

In conclusion, the increased portal diameter is associated with significantly increased risk for the development of PVT, and detection of portal diameter $>12.5 \mathrm{~mm}$ is highly sensitive for predicting PVT development. Additional studies are required to confirm the above results, identify additional risk factors in large sample size, and investigate the potential role of thromboprophylaxis for the suppression of PVT development in the high-risk population.

\section{Abbreviations}

PVT: portal vein thrombosis; CTA: computed tomography angiography; HBV: hepatitis B virus; ROC: receiver operating characteristic; OR: odds ratio; AUC: area under curve; OS: overall survival; MR: magnetic resonance.

\section{Declaration}

\section{Acknowledgements}

Not applicable.

\section{Authors' contributions}

SC conceived and designed the experiments. GD and XH collected and analyzed the clinical data and wrote the manuscript. $\mathrm{YZ}$ and $\mathrm{HD}$ operated ultrasonic devices and analysed the data. FL revised the manuscript. All authors read and approved the final manuscript. 


\section{Funding}

This study was supported by the fund of clinical research of Zhongshan Hospital, Fudan University (No. 2016ZSLC08) and partly supported by the Innovation Fund of Science and Technology Commission of Shanghai Municipality (No. 19411970200) and Shanghai Sailing Program (No. 19YF1406500).

\section{Availability of data and materials}

Data will be provided to those who have reasonable request by correspondence.

\section{Ethics approval and consent to participate}

This study protocol complied with the guidelines of the Declaration of Helsinki and was approved by the Medical Ethics Committee of Zhongshan Hospital, Fudan University, China.

\section{Consent for publication}

All authors agree with the submission and publication.

\section{Competing interests}

The authors declare no conflict of interest.

\section{References}

1.Amitrano L, Guardascione MA, Brancaccio V, Margaglione M, Manguso F, lannaccone L, et al. Risk factors and clinical presentation of portal vein thrombosis in patients with liver cirrhosis. JOURNAL OF HEPATOLOGY. 2004; 40:736-741.

2.Rodriguez-Castro KI, Porte RJ, Nadal E, Germani G, Burra P, Senzolo M. Management of nonneoplastic portal vein thrombosis in the setting of liver transplantation: a systematic review. TRANSPLANTATION. 2012; 94:1145-1153.

3.Stine JG, Shah PM, Cornella SL, Rudnick SR, Ghabril MS, Stukenborg GJ, et al. Portal vein thrombosis, mortality and hepatic decompensation in patients with cirrhosis: A meta-analysis. World J Hepatol. 2015; 7:2774-2780.

4.Stine JG, Pelletier SJ, Schmitt TM, Porte RJ, Northup PG. Pre-transplant portal vein thrombosis is an independent risk factor for graft loss due to hepatic artery thrombosis in liver transplant recipients. HPB (Oxford). 2016; 18:279-286. 
5.Shetty S, Ghosh K. Thrombophilic dimension of Budd chiari syndrome and portal venous thrombosis-a concise review. THROMBOSIS RESEARCH. 2011; 127:505-512.

6.Cagin YF, Bilgic Y, Berber I, Yildirim O, Erdogan MA, Firat F, et al. The risk factors of portal vein thrombosis in patients with liver cirrhosis. Experimental and Therapeutic Medicine. 2019;17:3189-3194.

7.Stine JG, Wang J, Shah PM, Argo CK, Intagliata N, Uflacker A, et al. Decreased portal vein velocity is predictive of the development of portal vein thrombosis: A matched case-control study. LIVER INTERNATIONAL. 2018; 38:94-101.

8.Qi X, Han G, He C, Yin Z, Guo W, Niu J, et al. CT features of non-malignant portal vein thrombosis: a pictorial review. Clin Res Hepatol Gastroenterol. 2012; 36:561-568.

9.Berzigotti A, Garcia-Criado A, Darnell A, Garcia-Pagan JC. Imaging in clinical decision-making for portal vein thrombosis. Nat Rev Gastroenterol Hepatol. 2014; 11:308-316.

10.Rector WJ, Campra J, Ralls PW, Charms M. Utility and limitations of splanchnic venous ultrasonography in diagnosis of portal hypertension. JOURNAL OF CLINICAL ULTRASOUND. 1986; 14:689-696.

11.Dong G, Huang X, Jiang S, Ni L, Chen S. Simvastatin Mitigates Apoptosis and Transforming Growth Factor-Beta Upregulation in Stretch-Induced Endothelial Cells. Oxidative Medicine and Cellular Longevity. 2019; 2019:6026051.

12.van Bommel F, Berg T. Treatment of HBV related cirrhosis. LIVER INTERNATIONAL. 2013; 33 SuppI $1: 176-181$.

13.Lertpipopmetha K, Auewarakul CU. High incidence of hepatitis B infection-associated cirrhosis and hepatocellular carcinoma in the Southeast Asian patients with portal vein thrombosis. BMC GASTROENTEROLOGY. 2011; 11:66.

14.Intagliata NM, Caldwell SH, Tripodi A. Diagnosis, Development, and Treatment of Portal Vein Thrombosis in Patients With and Without Cirrhosis. GASTROENTEROLOGY. 2019; 156:1582-1599.

15.Violi F, Lip GY, Cangemi R. Endotoxemia as a trigger of thrombosis in cirrhosis. HAEMATOLOGICA. 2016; 101:e162-e163.

16.Kinjo N, Kawanaka H, Akahoshi T, Matsumoto Y, Kamori M, Nagao Y, et al. Portal vein thrombosis in liver cirrhosis. World J Hepatol. 2014; 6:64-71.

17.Doggen CJ, Smith NL, Lemaitre RN, Heckbert SR, Rosendaal FR, Psaty BM. Serum lipid levels and the risk of venous thrombosis. Arterioscler Thromb Vasc Biol. 2004; 24:1970-1975. 
18.Girleanu I, Trifan A, Cojocariu C, Dimache M, Singeap AM, Stoica O, et al. Platelet indices in patients with de novo portal vein thrombosis and liver cirrhosis. Rev Med Chir Soc Med Nat lasi. 2013; 117:641647.

19.Mikula T, Kozlowska J, Stanczak W, Sapula M, Rozyk A, Wiercinska-Drapalo A. Serum ADAMTS-13 Levels as an Indicator of Portal Vein Thrombosis. Gastroenterol Res Pract. 2018; 2018:3287491.

20.Villa E, Camma C, Marietta M, Luongo M, Critelli R, Colopi S, et al. Enoxaparin prevents portal vein thrombosis and liver decompensation in patients with advanced cirrhosis. GASTROENTEROLOGY. 2012; 143:1253-1260.

21.Zhang DL, Hao JY, Yang N. Value of D-dimer and protein S for diagnosis of portal vein thrombosis in patients with liver cirrhosis. JOURNAL OF INTERNATIONAL MEDICAL RESEARCH. 2013; 41:664-672.

22.Zhang D, Hao J, Yang N. Protein C and D-dimer are related to portal vein thrombosis in patients with liver cirrhosis. J Gastroenterol Hepatol. 2010; 25:116-121.

23.Englesbe MJ, Kubus J, Muhammad W, Sonnenday CJ, Welling T, Punch JD, et al. Portal vein thrombosis and survival in patients with cirrhosis. Liver Transpl. 2010; 16:83-90.

\section{Figures}
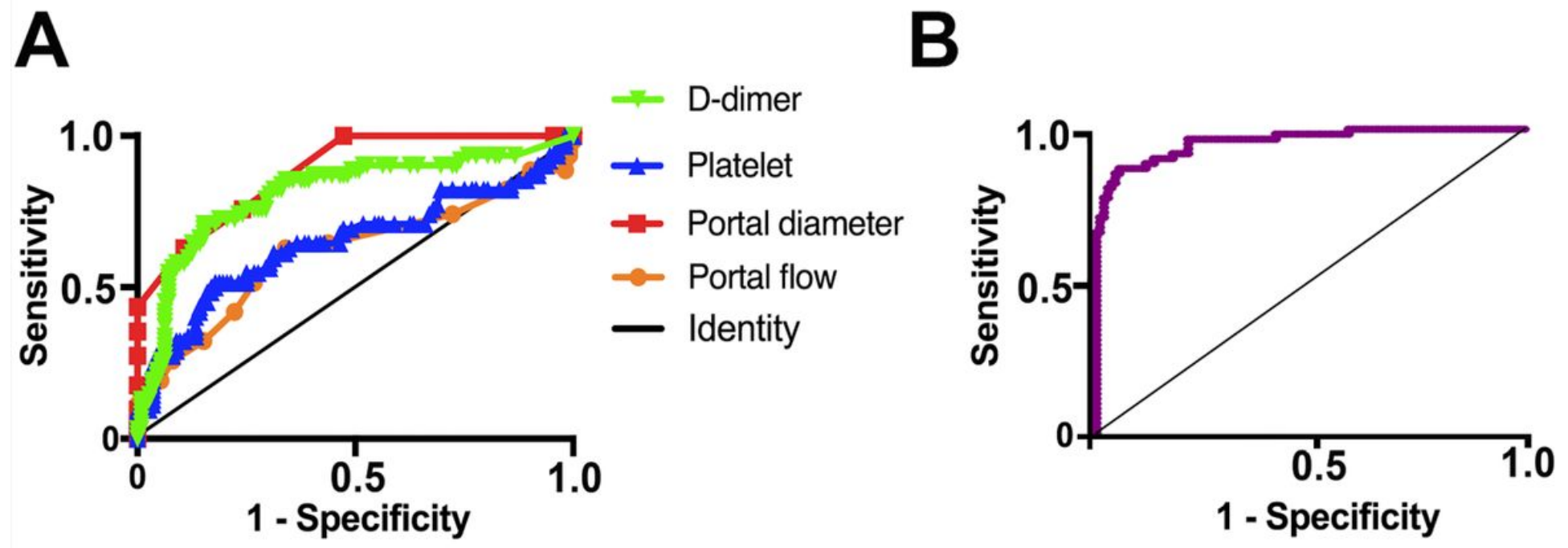

Figure 1

ROC analysis of portal diameter, portal flow, platelet count, D-dimer, and Child-Pugh score (A) or their combination (B) predicting the presence of PVT in cirrhotic patients. Portal diameter was the strongest independent risk factor predicting PVT development. 
10

20

30

40

50

60

70

80

90

100

Portal diameter

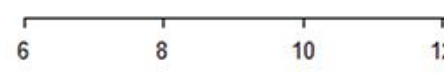

12

$14 \quad 16$

16

18

20

22

24

26

28

Portal flow

\begin{tabular}{lllll}
\hline 0.35 & 0.25 & 0.15 & 0.05
\end{tabular}

Platelet

\begin{tabular}{lllllll}
\hline 0 & 50 & 150 & 250 & 350 & 450
\end{tabular}

D-dimer

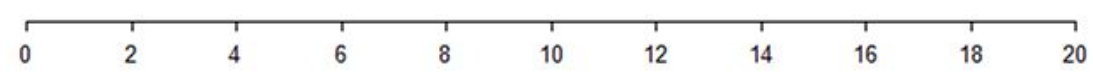

Total Points

\begin{tabular}{|c|c|c|c|c|c|c|c|}
\hline 0 & 20 & 40 & 60 & 80 & 100 & 120 & 140 \\
\hline
\end{tabular}

Risk

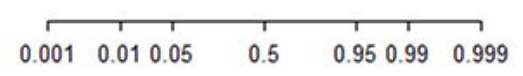

\section{Figure 2}

A nomograph for estimating the risk of portal diameter, portal flow, platelet count, and D-dimer in predicting PVT development. Portal diameter was the strongest independent risk factor predicting PVT development. 
A

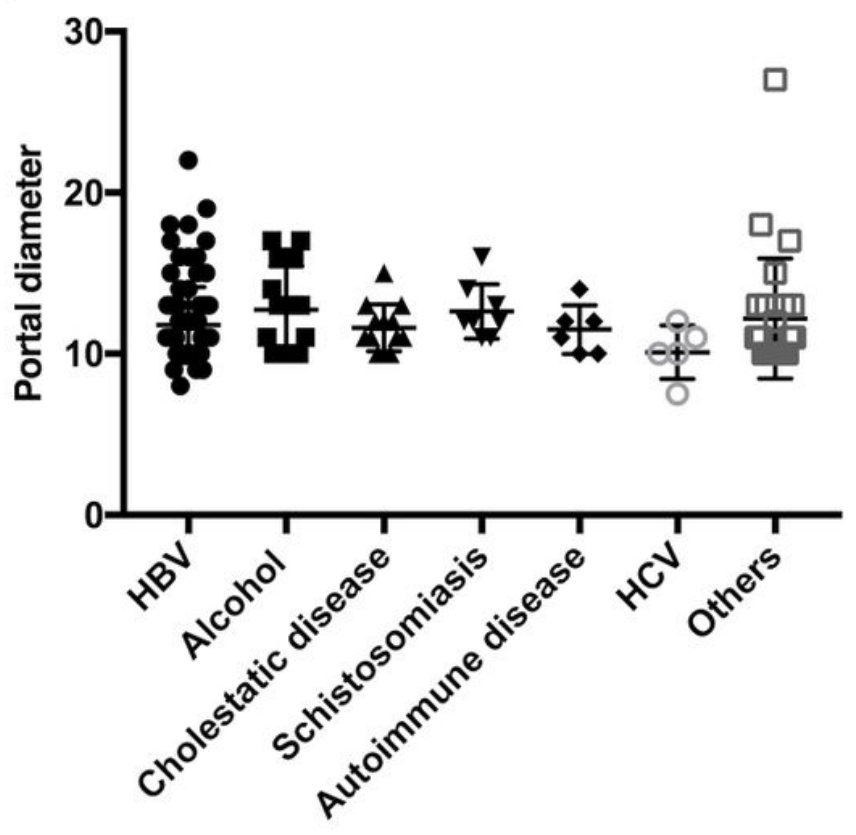

B

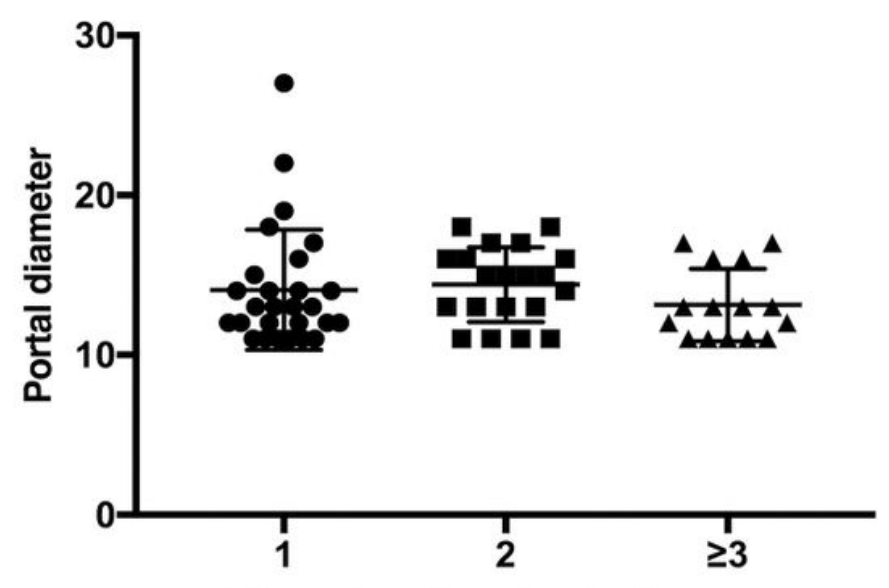

Thrombus involved sites

Figure 3

The effect of etiology (A) and the range of thrombus (B) on portal diameter. The range of thrombus included five sites: main portal vein (main PV), left portal vein (left PV), right portal vein (right PV), superior mesenteric vein, and splenic vein. Number of involved sites were divided into three groups: 1 (one site), 2 (two sites), $\geq 3$ (three sites).

A

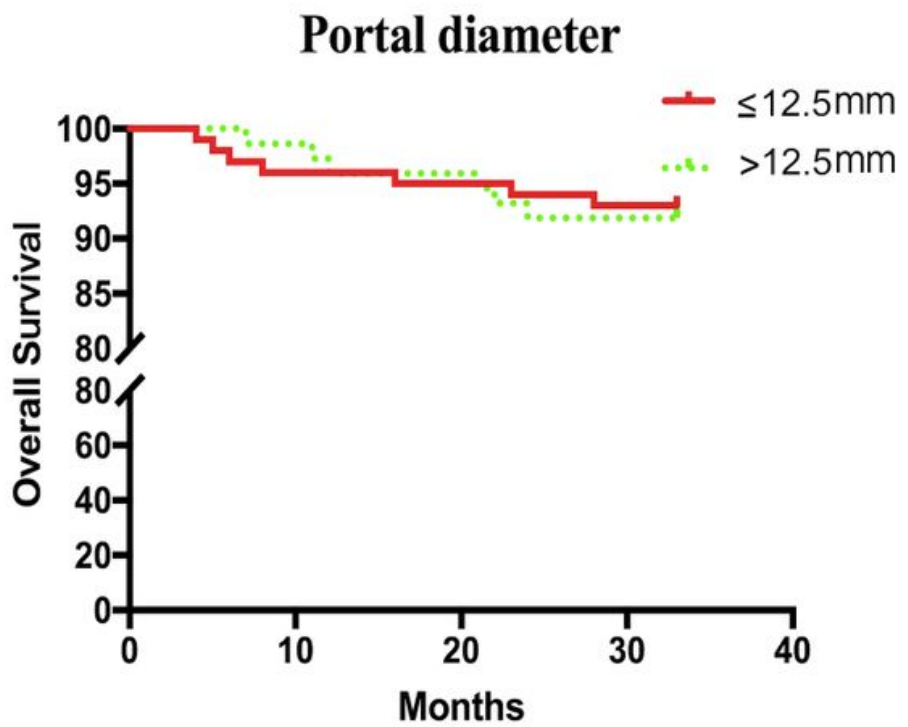

B

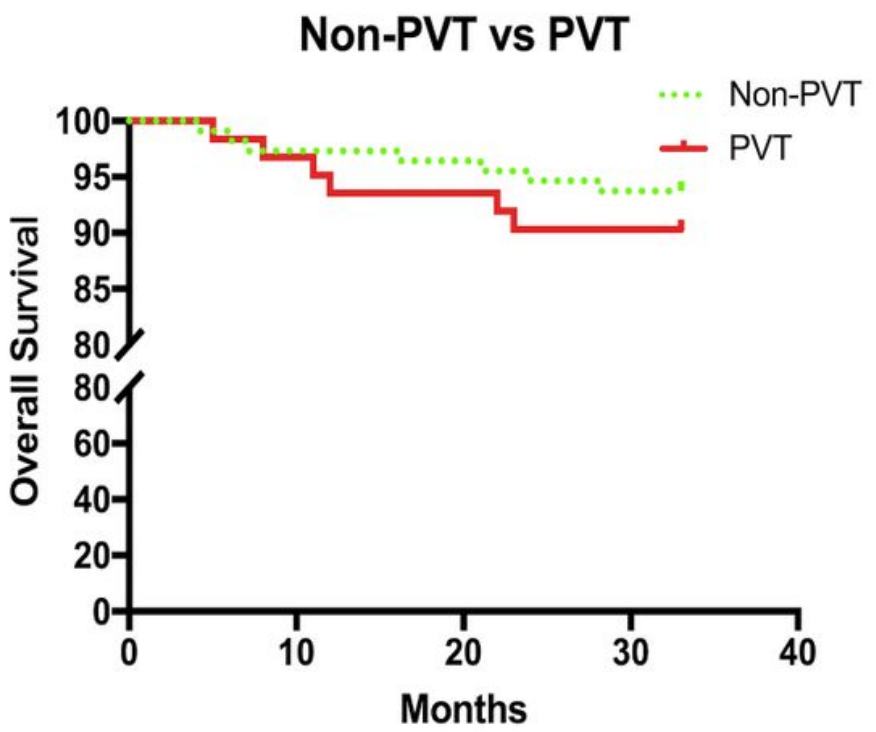

Figure 4

Kaplan-Meier OS curve was analyzed in patients with the stratified portal diameter with and without PVT. (A) No differences were observed in the OS when stratifying by portal diameter. (B) OS was similar in 
cirrhosis patients with and without PVT.

Page $12 / 12$ 